\title{
Dilemmas of Korean Athletes With a Spinal Cord Injury to Participate in Sports: A Survey Based on the ICF Core Set for Spinal Cord Injury
}

\author{
Je Shik Nam, MD ${ }^{1}$, Ko Eun Lee, MD $^{1}$, Ah Young Jun, MD $^{2}$, Chong Suck Parke, MD $^{3}$, \\ Hyun Young Kim, $\mathrm{MD}^{1}$, You Hyeon Chae, $\mathrm{MD}^{1}$ \\ ${ }^{1}$ Department of Rehabilitation Medicine, Bundang Jesaeng General Hospital, Seongnam; ${ }^{2}$ Department of Rehabilitation \\ Medicine, Ansan Workers' Compensation Hospital, Korea Workers' Compensation \& Welfare Service, Ansan; \\ ${ }^{3}$ Department of Orthopedic Surgery, Bundang Chuk Hospital, Seongnam, Korea
}

\begin{abstract}
Objective To explore the experiences of athletes with spinal cord injury (SCI) in Korea with respect to dilemmas of participating in sports with regards to the facilitators and barriers, using the International Classification of Functioning, Disability and Health (ICF).

Methods The facilitators and barriers to sports participation of individuals with SCI were examined using 112 ICF categories. A questionnaire in dichotomous scale was answered, which covered the subjects 'Body functions', 'Body structures,' 'Activity and participation' and 'Environmental factors'. Data analysis included the use of descriptive statistics to examine the frequency and magnitude of reported issues.

Results Sixty-two community-dwelling participants were recruited. Frequently addressed barriers in 'Body functions' were mobility related problems such as muscle and joint problems, bladder and bowel functions, pressure ulcers, and pain. In 'Activity and participation', most frequently reported were mobility and self-care problems. Highly addressed barriers in 'Environmental factors' were sports facilities, financial cost, transportation problems and lack of information. Relationships such as peer, family and friends were the most important facilitators.

Conclusion Numerous barriers still exist for SCI survivors to participate in sports, especially in the area of health care needs and environmental factors. Our results support the need for a multidisciplinary approach to promote sports participation.
\end{abstract}

Keywords Spinal cord injuries, Sports, Community participation

Received February 22, 2016; Accepted April 12, 2016

Corresponding author: Ko Eun Lee

Department of Rehabilitation Medicine, Bundang Jesaeng General Hospital, 20 Seohyeon-ro 180beon-gil, Bundang-gu, Seongnam 13590, Korea. Tel: +82-31-779-0063, Fax: +82-31-779-0635, E-mail: rhsrhs2u@gmail.com

ORCID: Je Shik Nam (http://orcid.org/0000-0003-4753-626X); Ko Eun Lee (http://orcid.org/0000-0002-3414-8121); Ah Young Jun (http://orcid. org/0000-0001-5783-5162); Chong Suck Parke (http://orcid.org/0000-0001-7083-2388); Hyun Young Kim (http://orcid.org/0000-0003-4920-8374); You Hyeon Chae (http://orcid.org/0000-0002-3403-0975).

(c) This is an open-access article distributed under the terms of the Creative Commons Attribution Non-Commercial License (http://creativecommons.org/ licenses/by-nc/4.0) which permits unrestricted noncommercial use, distribution, and reproduction in any medium, provided the original work is properly cited. Copyright $\odot 2016$ by Korean Academy of Rehabilitation Medicine 


\section{INTRODUCTION}

With an increase in the life expectancy for spinal cord injury (SCI) groups, interest in quality of life is also rising [1]. The ultimate goal of rehabilitation is achievement of an independent and healthy life. Promoting physical activity is assumed to reduce secondary impairment and chronic health problems, and it is also helpful for the psychological well-being [2]. Participation in sports activities means success of community-based rehabilitation, and this may help in the reduction of social cost such as medical care and loss of productivity [3].

Although there are expectations of several benefits, persons having SCI face many barriers to participate in physical activity. Loss of complex body functions, such as motor and sensory functions, autonomic regulations, bladder and bowel control, circulatory regulation and skin protection, restrict their sports participation [4]. Besides, there are systemic and environmental barriers such as accessibility, financial cost and information [5]. In Korea, environmental barriers such as lack of information and poor accessibility for the disabled add to the difficulties.

According to the results of research on needs of people with SCI in Korea 2012 , over $50 \%$ of respondents answered their willingness to use disability welfare facilities and disability gyms. Nearly $70 \%$ of respondents used private automobiles and about $30 \%$ of respondents got welfare service information from fellow people with SCI, as their primary methods of transportation [6]. Also, according to a 2015 survey on the participation of the disabled in physical activity in South Korea, only $4.3 \%$ of respondents used facilities for the disabled, and approximately half of the respondents ( $47 \%$ ) used a nearby park. This meant that systemic and environmental support level was lower than the needs.

In Korea, as part of an effort to enhance sports participation, we tried to identify barriers at the individual and the societal level for persons with SCI. We assessed functioning and disability in persons with SCI who are physically active, as per the International Classification of Functioning, Disability and Health (ICF) Core Set for SCI for long-term care [7].

\section{MATERIALS AND METHODS}

\section{Subjects}

This study involved questionnaire surveys of $62 \mathrm{com}$ munity-dwelling SCI athletes participating in sports activities. We visited sports clubs and distributed the questionnaire through a structured interview process that typically lasted 30 minutes. To be included in the interview, following criteria were required in the SCI patients: (1) living in a South Korean community, (2) having adequate communication function, and (3) regularly participating in sports activities at sports club for disabled. All participants provided their formal consent. The study was approved by the local Institutional Review Board and was carried out in accordance with the Declaration of Helsinki.

\section{Instrument development and measure}

We conducted qualitative interviews with 15 SCI persons regarding their sports activity experience after affliction with SCI, based on comprehensive ICF Core Set. The participants were asked to freely describe any limitations in participation. The presence of the barriers was noted for each of the following categories: 'Body functions', 'Body structures,' 'Activity and participation', and 'Environmental factors'. The dichotomous scale was used to indicate the presence or absence of a problem. The interview was conducted, and the data were analyzed by two physiatrists. We selected categories frequently answered in sample interviews for 'Body functions', and a questionnaire was made about specific problems using sublevel categories of each issue. For example, the category b735 'muscle tone functions' was assessed using additional question such as "Which body structures are the common barriers with muscle tone functions?". Responders were allowed to choose multiple answers in each question regarding sports activity, except for demographic purposes.

For the questionnaire on 'Body functions and structures', we referred to 7 chapters and 32 second level ICF categories regarding the core set of barriers. We also investigated the unmet medical care problems. In sample interviews, problems frequently answered were pain, urination, defecation, muscle tone, mobility of joint, and skin protection. Our questionnaire asked to identify the specific structures or functions about these problems us- 
ing third or fourth level ICF categories.

In 'Activity and participation components', the questionnaire was made using 9 chapters and 39 second level ICF categories. Sample interviews revealed a high prevalence with respect to mobility and self care.

In 'Environmental factors', the questionnaire was comprised from 5 chapters and 41 second level ICF categories. The sources of information such as type of sports and how to join the team were addressed. We also questioned the means of transportation used to get to sports clubs.

\section{Statistical analysis}

All analyses were performed using SPSS ver. 21.0 for Windows (IBM, Armonk, NY, USA). Data analysis included the use of descriptive statistics to examine the frequency and magnitude of reported barriers. All surveys were done in personal interview. There was no missing data.

\section{RESULTS}

\section{Sociodemographic data}

Table 1 shows the sample characteristics. A total of 62 community-dwelling SCI individuals (85.5\% men, mean age $42.8 \pm 13.5$ years and mean onset duration $13.0 \pm 11.1$ years) were recruited. Time until start of sports activity after SCI was $4.5 \pm 2.3$ years. Participation duration was $5.2 \pm 4.1$ years. Fifty-two participants $(83.9 \%)$ had a high school education, and marital status of 38 individuals (61.3\%) was single. Thirty-two participants (51.6\%) reported having a job. Forty participants $(67.7 \%)$ had complete lesions spinal cord lesions, and $53(85.5 \%)$ had paraplegia. The types of sports participated were wheelchair rugby (46.8\%), lawn bowling $(45.2 \%)$, and wheelchair basketball (8.1\%).

\section{Functioning and disability}

Table 2 shows the ICF categories over $40 \%$ in persons with SCI. For 'Body functions', 10 of the 32 ICF second level categories were documented over $40 \%$ in the respondents. Majority of the participants mentioned ' $b 7$ Neuromusculoskeletal and movement-related functions', such as spasticity, weakness, joint contracture and loss of movement sense. We identified specific structures or functions regarding these issues using sublevel categories (Table 3). 'b735 Muscle tone dysfunctions' had the highest prevalence in the 'Body function and structure' com- ponent. For this category, 's75002 Muscle of thigh' and 's75012 Muscle of lower leg' were highly addressed. Other common categories were 'b620 Urination functions', 'b525 Defecation functions,' 'b810 Protective functions of the skin' and 'b280 Sensation of pain' showing a prevalence exceeding 55\%. Regarding unmet medical care problems, highly placed issues were bladder functions, pain, spasticity and pressure sores. There were different tendencies between barriers and unmet medical needs. Some participants mentioned the following regarding medical needs:

Table 1. General characteristics of subjects $(n=62)$

\begin{tabular}{|cc|}
\hline \multicolumn{1}{|c}{ Characteristic } & Value \\
\hline Age (yr) & $42.8 \pm 13.5$ \\
\hline Sex & $53(85.5)$ \\
\hline Male & $9(14.5)$ \\
\hline Female & $13.0 \pm 11.1$ \\
\hline Onset duration (yr) & \\
\hline Level of injury & $9(14.5)$ \\
\hline Tetraplegia & $53(85.5)$ \\
\hline Paraplegia & \\
\hline Completeness of injury & $40(67.7)$ \\
\hline Complete & $22(35.5)$ \\
\hline Incomplete & $4.5 \pm 2.3$ \\
\hline Time until to start sports activity after & \\
injury (yr) & $5.2 \pm 4.1$ \\
\hline Participation duration (yr) & \\
\hline Post-injury employment status & $32(51.6)$ \\
\hline Working & $30(48.4)$ \\
\hline Not working & \\
\hline Highest level of education completed & $2(3.2)$ \\
\hline Elementary school & $8(12.9)$ \\
\hline Middle school & $30(48.4)$ \\
\hline High school & $22(35.5)$ \\
\hline University of college & $38(61.3)$ \\
\hline Marital status & $24(38.7)$ \\
\hline Single & $29(46.8)$ \\
\hline Married & $28(45.2)$ \\
\hline Sports type & $5(8.1)$ \\
\hline Wheelchair rugby & \\
\hline Lawn bowling & \\
\hline Wheelchair basketball & \\
\hline
\end{tabular}

Values are presented as mean \pm standard deviation or number (\%). 
Table 2. The frequencies of ICF categories mentioned over $40 \%$ in the participants $(n=62)$

\begin{tabular}{|c|c|c|}
\hline ICF code & ICF category title & No. of participants (\%) \\
\hline \multicolumn{3}{|c|}{ Body functions barriers - chapters } \\
\hline b7 & Neuromusculoskeletal and movement-related functions & $55(89)$ \\
\hline b6 & Genitourinary and reproductive functions & $50(81)$ \\
\hline b5 & Functions of the digestive, metabolic and endocrine systems & $42(68)$ \\
\hline b8 & Functions of the skin and related structures & $39(63)$ \\
\hline b2 & Sensory functions and pain & $34(55)$ \\
\hline \multicolumn{3}{|c|}{ Body functions barriers - second level ICF categories } \\
\hline b735 & Muscle tone functions & $49(79)$ \\
\hline b620 & Urination functions & $47(76)$ \\
\hline b730 & Muscle power functions & $43(69)$ \\
\hline b525 & Defecation functions & $42(68)$ \\
\hline b810 & Protective functions of the skin & $39(63)$ \\
\hline b280 & Sensation of pain & $34(55)$ \\
\hline b710 & Mobility of joint functions & $32(52)$ \\
\hline b740 & Muscle Endurance functions & $30(48)$ \\
\hline b780 & Sensations related to muscle and movement functions & $29(47)$ \\
\hline b270 & Sensory functions related to temperature and other stimuli & $26(42)$ \\
\hline \multicolumn{3}{|c|}{ Body functions - unmet medical care problems } \\
\hline b620 & Urination functions & $37(60)$ \\
\hline b280 & Sensation of pain & $33(53)$ \\
\hline b735 & Muscle tone functions & $30(48)$ \\
\hline b810 & Protective functions of the skin & $26(42)$ \\
\hline \multicolumn{3}{|c|}{ Activity and participation barriers - chapters } \\
\hline $\mathrm{d} 4$ & Mobility & $50(81)$ \\
\hline d5 & Self care & $37(60)$ \\
\hline d8 & Major life areas & $28(45)$ \\
\hline \multicolumn{3}{|c|}{ Activity and participation barriers - second level ICF categories } \\
\hline $\mathrm{d} 415$ & Maintaining a body position & $40(65)$ \\
\hline $\mathrm{d} 410$ & Changing basic body position & $38(61)$ \\
\hline $\mathrm{d} 420$ & Transferring oneself & $34(55)$ \\
\hline $\mathrm{d} 445$ & Hand and arm use & $32(52)$ \\
\hline $\mathrm{d} 440$ & Fine hand use & $30(48)$ \\
\hline $\mathrm{d} 510$ & Washing oneself & $30(48)$ \\
\hline d530 & Toileting & $28(45)$ \\
\hline d850 & Remunerative employment & $28(45)$ \\
\hline d520 & Caring for body parts & $27(44)$ \\
\hline $\mathrm{d} 540$ & Dressing & $26(42)$ \\
\hline \multicolumn{3}{|c|}{ Environmental factors barriers - chapters } \\
\hline el & Products and technology & $50(81)$ \\
\hline e5 & Services, system and polices & $42(68)$ \\
\hline $\mathrm{e} 4$ & Attitudes & $30(48)$ \\
\hline
\end{tabular}


Table 2. Continued

\begin{tabular}{|c|c|c|}
\hline ICF code & ICF category title & No. of participants (\%) \\
\hline \multicolumn{3}{|c|}{ Environmental factors barriers - second level ICF categories } \\
\hline e150 & $\begin{array}{l}\text { Design, construction and building products and technology of } \\
\text { buildings for public use }\end{array}$ & $45(73)$ \\
\hline el40 & Products and technology for culture, recreation and sports & $38(61)$ \\
\hline e165 & Assets & $36(58)$ \\
\hline e540 & Transportation services, systems and policies & $34(55)$ \\
\hline e590 & Labour and employment services, systems and polices & $33(53)$ \\
\hline e575 & General social support services, systems and policies & $32(52)$ \\
\hline e430 & Individual attitudes of people in positions of authority & $25(40)$ \\
\hline \multicolumn{3}{|c|}{ Environmental factors facilitators - chapters } \\
\hline e3 & Support and relationships & $55(89)$ \\
\hline e4 & Attitudes & $45(73)$ \\
\hline el & Products and technology & $40(65)$ \\
\hline \multicolumn{3}{|c|}{ Environmental factors facilitators - second level ICF categories } \\
\hline e325 & Acquaintances, peers, colleagues, neighbors and community members & $44(71)$ \\
\hline e310 & Immediate family & $40(65)$ \\
\hline e320 & Friends & $38(61)$ \\
\hline e425 & $\begin{array}{l}\text { Individual attitudes of acquaintances, peers, colleagues, neighbors } \\
\text { and community members }\end{array}$ & $37(60)$ \\
\hline el25 & Products and technology for communication & $36(58)$ \\
\hline e110 & Products or substances for personal consumption & $35(56)$ \\
\hline e450 & Individual attitudes of health professionals & $32(52)$ \\
\hline el40 & Products and technology for culture, recreation and sport & $27(44)$ \\
\hline e120 & $\begin{array}{l}\text { Products and technology for personal indoor and outdoor mobility } \\
\text { and transportation }\end{array}$ & $26(42)$ \\
\hline
\end{tabular}

ICF, International Classification of Functioning, Disability and Health.

"It's impossible to get a help for weakness and sensory loss with medication. Urinary incontinence and muscle spasm are major problems related to movement. These problems are not well controlled with medication."

For 'Activities and participation', 'd4 Mobility' and 'd5 Self care' were frequently mentioned at the chapter level. Ten of the 39 ICF second level categories were more than $40 \%$ in the respondents. Majority of the SCI respondents complained of balance issues, such as ' $\mathrm{d} 415$ Maintaining a body position' and ' $\mathrm{d} 410$ Changing basic body position'. Regarding 'd8 Major life areas', only 'd850 Remunerative employment' were identified more than $45 \%$.

\section{Environmental factors}

In 'Environmental factors', 7 categories as a barrier and 9 categories as a facilitator of the 41 ICF second level categories were over $40 \%$ in the respondents. As barrier factors, 'e1 Products and technology,' 'e5 Services, system and polices', and 'e4 Attitudes', were most frequently addressed at the chapter level. The building design, transportation and economic issues were main problems. As facilitators, 'e3 Support and relationships,' 'e4 Attitudes' and 'el Products and technology' were commonly reported at the chapter level. Relationships with peers, family and friends were the most important facilitators.

Transportations were experienced both as a barrier as well as a facilitator. Most participants used owner driven car when going to sports club (Table 4). Some respondent mentioned the following about these problems:

"Most of the SCI people have to drive their own car, since a means of public transportation doesn't have enough room for carrying sports equipment and wheelchairs. In fact, unless you are wealthy enough 
Table 3. Body function and structure subset assessment for sports participation (top 3 rated)

\begin{tabular}{|c|c|c|}
\hline ICF code & ICF category title & $\begin{array}{c}\text { No. of } \\
\text { participants } \\
\text { (\%) }\end{array}$ \\
\hline b735 Muscle tone functions (n=49) & \\
\hline s75002 & Muscles of thigh & $32(65)$ \\
\hline s75012 & Muscles of lower leg & $26(53)$ \\
\hline s7202 & Muscles of shoulder region & $25(51)$ \\
\hline b620 Urination functions (n=47) & \\
\hline b6200 & Urination & $40(85)$ \\
\hline b6202 & Urinary continence & $25(53)$ \\
\hline b6201 & Frequency of urination & $20(43)$ \\
\hline b730 Muscle power functions (n=43) & $33(77)$ \\
\hline s7601 & Muscles of trunk & $30(70)$ \\
\hline s73002 & Muscle of upper arm & $27(63)$ \\
\hline s7202 & Muscles of shoulder region \\
\hline b525 Defecation functions (n=42) & \\
\hline b5250 & Elimination of faeces & $38(90)$ \\
\hline b5251 & Faecal consistency & $28(67)$ \\
\hline b5252 & Frequency of defecation & $27(64)$ \\
\hline b810 Protective function of the skin (n=39) \\
\hline s8103 & Skin of pelvic region & $25(64)$ \\
\hline s8104 & Skin of lower extremity & $20(51)$ \\
\hline s8102 & Skin of upper extremity & $13(33)$ \\
\hline b280 Sensation of pain (n=34) & \\
\hline b28014 & Pain in upper limb & $25(73)$ \\
\hline b28015 & Pain in lower limb & $24(70)$ \\
\hline b28013 & Pain in back & $20(59)$ \\
\hline b710 Mobility of joint functions (n=32) & \\
\hline s75021 & Ankle joint and joints of & $24(75)$ \\
\hline s7201 & foot and toes & Joints of shoulder region \\
\hline s73021 & Joint of hand and fingers & $16(50)$ \\
\hline
\end{tabular}

ICF, International Classification of Functioning, Disability and Health.

to afford the activities, it is impossible to do so because you actually have to purchase your own sports equipment, such as sports wheelchairs and a car". "When we have games, we have to travel by our own car. Our team doesn't have a bus for people with disability. Near the stadium, some accommodations don't have an elevator, and the toilet is too small to get in by wheelchair."

They answered that after SCI, family members and
Table 4. Route and transportation for sports activities participation $(\mathrm{n}=62)$

\begin{tabular}{lc}
\hline & $\begin{array}{c}\text { No. of } \\
\text { participants (\%) }\end{array}$ \\
\hline How to get information about sports & \\
Acquaintance & $45(73)$ \\
\hline Internet & $8(13)$ \\
Social worker & $7(11)$ \\
Health care provider & $2(3)$ \\
Means of transportation & \\
Owner-driven car & $59(95)$ \\
Public transportation & $2(3)$ \\
Taxi for disabled & $1(2)$ \\
\hline
\end{tabular}

friends are more supportive rather than the medical teams or health experts. Most common source of information to participate in sports activity is through the individuals with SCI or internet.

"Even though I want to participate in sports activities, I don't know where and how to exercise, and the physical consequences. My doctor never talked to me about sports activity. I got information from wheelchair salesman."

\section{DISCUSSION}

In America or Europe, many studies have reported barriers of sports participation in people with SCI $[8,9]$. These studies were reported in developed countries [10], where there may be cultural differences and thus might not be relevant for the Korean environment. Compared to other concerns with SCI groups, studies related to sports participation with SCI patients are relatively limited in South Korea. Based on our best knowledge, there have been some studies that assessed effects of sports participation, such as quality of life or disability acceptance $[11,12]$. But suggestions on how to promote sports participation was lacking.

In our studies, we obtained a more comprehensive understanding of the current issues of sports participation with SCI athletes in South Korea, by using the ICF Core Set for SCI for long-term care; we presented the data suitable for the current situation in South Korea. Previous studies have been very diverse in terms of study outcomes and data reporting. Comparing other health status measures, the ICF as a part of the World Health 
Organization's international family of classifications, is a contemporary framework to harmonize the assessment of functioning and disability at the individual and the societal level. Also, there has been confusion on the definition of participation; many health literatures have used varied terms such as participation, social participation and community participation. The ICF domains of 'Activity and participation' considered to clarify these definitions of social and community participation are 'Interpersonal interactions and relationships,' 'Major life areas', and 'Community, social, and civic life' [13]. However, sports are only a part of the broader definition of physical activity or community participation. They can be defined in many ways, from social connectedness with other individuals to intensive sports activity. Therefore, we focused more on sports participation, including community-dwelling SCI athletes groups.

The frequently addressed impairments in component 'Body functions' were movement related functions, bladder and bowel functions, pain sense and pressure ulcer. These problems are well-known complication of SCI and supported by previous studies [14].

Movement related functions such as muscle power and tone function, are important factors for sports performance. Most SCI persons answered the major issues were muscle strengthening rather than motor recovery. They also thought the muscle tone functions such as control of spasticity were important factors in participating for sports activity. Regarding muscle tone and joint mobility dysfunctions, problems of the lower extremity were frequently addressed. The change of skeletal muscle fiber types in SCI can affect aerobic capacity [15], but in this study, few respondents checked 'b455 exercise tolerance functions. These findings may be related to the higher number of people with paraplegia and a fewer with tetraplegia in this study.

Bladder and bowel dysfunctions were reported to be common problems. We identified these problems with third level ICF categories. Lack of bladder and bowel autonomy was more addressed than incontinence. These problems are associated with low levels of physical quality of life domains and high mortality [16]. Several studies reported an increasing prevalence of cardiovascular and thermoregulatory problems with time after SCI $[17,18]$. Some quadriplegia participants reported being challenged by managing this problem during sports activities.
These problems were not highly checked, because almost all participants were paraplegics in this study.

Skin problems were frequently mentioned by the participants. Prevalence of pressure sore for persons with chronic SCI varied between $15 \%$ and $30 \%$ [19].

In our study, about $70 \%$ of responders reported experiencing pressure sore since sports participation. This finding may be related to direct soft tissue trauma of lower extremity and longer duration of wheelchair seating time during sports activities.

The participants needed maximum medical care for 'urination functions' (b620) and 'sensation of pain' (b280). These findings showed discrepancy between barrier factors and needs for care, when related with sports participation. Most of the SCI responders were on medication for the management of bladder, bowel, pain and spasticity issues. However, they still had unmet problems such as urinary incontinence and musculoskeletal pain. We also addressed the 'sensation of pain' (b280) with fourth level categories. Frequently addressed body structures were upper limb, lower limb and back. Taylor and Williams [20] reported the prevalence of sports related injury among the wheelchair racing athletes was $72 \%$ during a 12 -month period, with upper extremity injuries being the maximum. Our study did not cover the reason of pain or mechanism of injury; overuse injuries have also been identified as important mechanisms. For preventing sports injuries, injury risk factors and mechanisms could be established. Along with preventive strategies, training programs should be provided with medical support. Physicians should be aware of this need and consider a specialized care related with activity and participation during the treatment of SCI.

The most often mentioned barriers of activity and participation were 'mobility' (d4) and 'self care' (d5). Regarding mobility issues, maintaining and changing body positions, transfer and hand and arm use were frequently reported problems. In dynamic situations like sport activities, balance and stabilization capability are major concern. For preparing sports participation, balance and hand use training under dynamic stress should be considered during rehabilitation therapy [21].

A large number of 'Environmental factors' were included in the comprehensive ICF Core Set. 'Environmental factors' are important for assessing problems and planning the transition from hospital to the community in 
people with SCI. The frequently addressed barriers were sports facilities, financial cost, transportation problems and lack of information.

Limited sports facility was one of the problems. There are few sports club for disabled in Korea, and accessibility to public fitness facilities is limited because of barriers such as lack of ramp, stairs, narrow doorway, narrow toilet and inaccessible shower rooms. In case of team sports, finding inexpensive accommodation having a facility for the disabled and situated near the stadium is difficult during the competition season.

Exercise cost was identified frequently as barrier and facilitator. Purchasing and repairing wheelchair for sports is expensive, and there is not enough funding to support disabled sports activity. Using public transportation to get to exercise facilities is hard, therefore having own car is absolutely essential to access facilities and to carry sports equipment. Almost all of our participants had their own car and sports equipment, which they mentioned as a facilitator. But for overall disabled groups, economic issues could be a barrier to engage in physical activity. Public transportation with reasonable wheelchair lift, accessible doors and curbs should be considered in a public health policy especially in the developing countries. Improving accessibility to sports facilities will encourage individuals with SCI to engage in community programs.

According to a survey on physical activity of people with disability in Korea, over half the respondents reported they needed professional support for sports activity, and around $20 \%$ of respondents had help from professional physical coach at the public sports facility [22]. There are few specialized supports for disability sport in Korea. In some advanced countries, there are national organizations to provide individualized information and develop guidelines about disability sports, such as Active Living Alliance for Canadians with a Disability, and the National Center on Health, Physical Activity and Disability of America. They also refer people with disability to community transformation programs through a network.

Although there is a lack of professional support, the relationship with peer, family and friends were reported as a facilitator. One purpose of participating in sports was to make social contacts. Several SCI participants relied on interacting with their peers with disabilities. They also got the information from the peers about accessible facilities, sports skills and even managing medical problems such as overuse injury. Before returning to the community, SCI patients have to be supported to find the type of sports they could enjoy. As a part of health and social services, community based rehabilitation programs have to provide information, financial support and health counseling about sports activities. Health professionals have to consider how to promote the physical activity of SCI patients, and inform them where and how to exercise.

This study had some limitations. First, the study was conducted on SCI athletes in sports clubs such as basketball, lawn bowling and rugby team. The findings may not be generalized to all types of sports activity. Our participants are likely to be physically more active and in higher level of economic status among disabled groups. Second, the subjects were small in number. Besides, almost all subjects were paraplegics. There might be limitations in applying our research findings to all SCI patients. Third, the ICF was applied in a restricted way in this study. Respondents answered the questions in dichotomous scale indicating only the presence or absence of a problem, instead of in a five step scale. Fourth, although we did not evaluate the personal factor separately, we thought these included the body function categories such as health problem, lack of energy and fatigue. However, regarding pre-injury exercise experiences, some participants addressed this issue as a facilitator. This is known as one of predictor active post-injury sports participation. We could not link that to appropriate ICF categories of the Core Set.

We explored the experiences of athletes with SCI in South Korea with respect to dilemmas of sports participation, especially regarding the facilitators and barriers to sports participations using the ICF Core Set. The results showed that the ICF language in general covers a broad range of the individual experiences of persons with SCI. Identified function, disability and environmental issues showed some differences compared with common problems with SCI. Thus, we suggest considering in making an ICF Core Set for specific participation such as sports activity. Rehabilitation professionals can use this core set as a guideline to meet the needs of individuals with SCI. Also, it will be useful to promote community integration for SCI through a multidisciplinary approach. 


\section{CONFLICT OF INTEREST}

No potential conflict of interest relevant to this article was reported.

\section{REFERENCES}

1. Garshick E, Kelley A, Cohen SA, Garrison A, Tun CG, Gagnon D, et al. A prospective assessment of mortality in chronic spinal cord injury. Spinal Cord 2005;43:40816.

2. Nash MS. Exercise as a health-promoting activity following spinal cord injury. J Neurol Phys Ther 2005; 29:87-103, 106.

3. Forchheimer M, Tate DG. Enhancing community reintegration following spinal cord injury. NeuroRehabilitation 2004;19:103-13.

4. McKinley WO, Jackson AB, Cardenas DD, DeVivo MJ. Long-term medical complications after traumatic spinal cord injury: a regional model systems analysis. Arch Phys Med Rehabil 1999;80:1402-10.

5. Rimmer JH, Riley B, Wang E, Rauworth A, Jurkowski J. Physical activity participation among persons with disabilities: barriers and facilitators. Am J Prev Med 2004;26:419-25.

6. Korea Spinal Cord Injury Association. The results of the research on needs and actual conditions of people with spinal cord injury in Korea. Seoul: Korea Spinal Cord Injury Association; 2012.

7. Cieza A, Kirchberger I, Biering-Sorensen F, Baumberger M, Charlifue S, Post MW, et al. ICF Core Sets for individuals with spinal cord injury in the longterm context. Spinal Cord 2010;48:305-12.

8. Scelza WM, Kalpakjian CZ, Zemper ED, Tate DG. Perceived barriers to exercise in people with spinal cord injury. Am J Phys Med Rehabil 2005;84:576-83.

9. Tasiemski T, Kennedy P, Gardner BP, Blaikley RA. Athletic identity and sports participation in people with spinal cord injury. Adapt Phys Activ Q 2004;21:364-78.

10. Barclay L, McDonald R, Lentin P. Social and community participation following spinal cord injury: a critical review. Int J Rehabil Res 2015;38:1-19.

11. Kim CG, Lee HS. Effect of rehabilitation sports program on self-efficacy, sport understanding and sport participation in spinal cord injury persons. J Sport Leis Stud 2003;20:773-81.

12. Lee MS, Langsner SJ. Effects of participation in sport activity on social support and disability acceptance in individuals with spinal cord injury. Korean J Adapt Phys Activ 2011;19:91-102.

13. Chang FH, Coster WJ, Helfrich CA. Community participation measures for people with disabilities: a systematic review of content from an international classification of functioning, disability and health perspective. Arch Phys Med Rehabil 2013;94:771-81.

14. Pagliacci MC, Franceschini M, Di Clemente B, Agosti M, Spizzichino L; GISEM. A multicentre follow-up of clinical aspects of traumatic spinal cord injury. Spinal Cord 2007;45:404-10.

15. Grimby G, Broberg C, Krotkiewska I, Krotkiewski M. Muscle fiber composition in patients with traumatic cord lesion. Scand J Rehabil Med 1976;8:37-42.

16. Liu CW, Huang CC, Yang YH, Chen SC, Weng MC, Huang MH. Relationship between neurogenic bowel dysfunction and health-related quality of life in persons with spinal cord injury. J Rehabil Med 2009;41: 35-40.

17. Price MJ. Thermoregulation during exercise in individuals with spinal cord injuries. Sports Med 2006; 36:863-79.

18. Phillips WT, Kiratli BJ, Sarkarati M, Weraarchakul G, Myers J, Franklin BA, et al. Effect of spinal cord injury on the heart and cardiovascular fitness. Curr Probl Cardiol 1998;23:641-716.

19. Gelis A, Dupeyron A, Legros P, Benaim C, Pelissier J, Fattal C. Pressure ulcer risk factors in persons with spinal cord injury part 2: the chronic stage. Spinal Cord 2009;47:651-61.

20. Taylor D, Williams T. Sports injuries in athletes with disabilities: wheelchair racing. Paraplegia 1995; 33:296-9.

21. Bernard PL, Peruchon E, Micallef JP, Hertog C, Rabischong P. Balance and stabilization capability of paraplegic wheelchair athletes. J Rehabil Res Dev 1994;31:287-96.

22. Korea Ministry of Culture, Sports and Tourism. Survey on physical activity of people with disability in Korea. Sejong: Ministry of Culture, Sports and Tourism; 2013. 